\title{
INDUSTRIE MUSICALE ET (EN)JEUX D'ÉCHELLES : \\ LES PASSAGES DU LOCAL AU GLOBAL DANS \\ LA « LATIN MUSIC " PRODUITE À MIAMI
}

\author{
MUSIC INDUSTRY AND SCALE ISSUES: \\ FROM LOCAL TO GLOBAL IN \\ THE MIAMI'S LATIN MUSIC PRODUCTION
}

\author{
Alix Bénistant*
}

\section{Introduction}

Dès les années 1980, des chercheurs de diverses parties du monde tels qu'André Lange (1986), Terry Bright (1986), Dave Laing (1986), Nestor García Canclini (1988) ou Robert Burnett (1989) se sont intéressés à l'industrie du disque et aux stratégies mises en place dans l'articulation du local et du global. L'objectif était non seulement d'observer les circulations des contenus musicaux à différentes échelles, mais également de voir quelles étaient les esthétiques parvenant à la plus large diffusion, et celles confinées à une diffusion locale. Ces travaux souhaitaient ainsi mettre en évidence le déséquilibre des flux culturels à l'échelle mondiale. Dans cette perspective, nous souhaitons replacer les logiques de création d'une culture populaire "latino " ${ }^{1}$ faite d'appropriations et emprunts à des répertoires et identités multiples, au sein d'un contexte global où les phénomènes de concentration et de domination mis en place par l'industrie sont déterminants.

En ce sens, l'industrie musicale installée dans la ville floridienne pour gérer ce marché latino, observée attentivement au cours

\footnotetext{
* Docteur en sciences de l'information et de la communication (CEMTI). Université Paris 8 (Saint-Denis/ FR). alix.benistant@gmail.com.

1. Nous utilisons volontairement le terme " latino " de façon invariable et en abrégé. Il nous sert à rendre compte de multiples réalités telles qu'elles se retrouvent dans les discours des acteurs dominants du secteur musical autour d'une construction homogénéisante des marchés, musiques et publics à la fois latinoaméricains et latino-étasuniens. Ainsi, pour le distinguer de la catégorie ethnique du recensement nordaméricain, qui ne prend en compte que les individus vivant aux États-Unis se considérant comme " Latinos ", le terme utilisé en ce sens apparaîtra toujours en italique.
} 
d'un travail doctoral inscrit dans le champ des sciences de l'information et de la communication soutenu en 2016 à l'Université Paris 8 , s'avère être un objet pertinent pour observer la mise en circulation de contenus culturels réifiés dans un contexte de mondialisation économique et culturelle. La focalisation sur celui-ci, en mobilisant les outils proposés par l'économie politique de la communication - de laquelle découle la théorie des industries culturelles en France (MIĖGE, 2012) -, autorise à réinscrire des stratégies marchandes (largement étudiées), au sein d'un environnement politique, économique et social historiquement situé, quant à lui peu observé dans ses interrelations avec les logiques industrielles.

Pour parvenir à cela, nous allons procéder en trois temps. Seront tout d'abord explicités les cadres théorique et méthodologique adoptés pour appréhender ces phénomènes. Nous reviendrons notamment sur trois études pionnières ayant analysé le fonctionnement de l'industrie musicale internationale dans une perspective socioéconomique critique. L'enjeu est d'exposer les cadres d'interprétation mobilisés sur notre propre terrain miaméen, qui sera, quant à lui, introduit dans une deuxième partie contextuelle : quelle place les industries culturelles de Miami occupent dans ces circulations locales, régionales et internationales ? Enfın, notre terminerons avec la présentation de la stratégie multiscalaire mise en place par l'industrie musicale de Miami pour atteindre des audiences toujours plus étendues et des publics toujours plus divers.

Ce travail s'appuie principalement - outre des entretiens semi-directifs menés auprès de professionnels du secteur en 2010 et 2014 sur les données recueillies lors d'une analyse systématique de la presse professionnelle, à travers le magazine Billboard et sa rubrique clé pour notre sujet : Latin Notas. Celle-ci apparaît le 3 juillet 1982 " en réponse à la forte expansion et à l'influence croissante de la musique en langue espagnole ", souligne l'éditorial du numéro. Face à la diffıculté de récolter des données industrielles (ce que soulève André Lange dès $1986^{2}$ ), nous avons tenté, via cette source principale, de reconstituer les différentes étapes de la construction du marché latino par l'industrie dominante, en gardant toujours à l'esprit le fait que celui-ci reste bien un " construit social ", à l'instar des statistiques et chiffres avancés dans ce type de média (MATTHEWS, PERTICOZ: p. 14), avant tout tribune pour les professionnels du secteur.

\section{L'industrie musicale au cœur d'enjeux globaux}

Le secteur musical, qui trouve à Miami un " point territorial de référence pour la transnationalisation de son industrie " (MATO,

2. "Des difficultés méthodologiques sont diffıcilement surmontées, en particulier en ce qui concerne la récolte systématique de données scientifiquement construites. La dépendance est grande à l'égard des entreprises privées et des institutions publiques : il est fréquent que les données importantes soient tenues confidentielles, pour des raisons professionnelles ou fiscales, que leur mode de récolte ne soit pas vérifiable et que des séries statistiques diachroniques (bilans d'entreprises, répartition du chiffre d'affaires entre diverses activités, courbes de vie des produits, etc.) ne puissent être constituées. Ces données faisant souvent défaut, alors qu'elles sont les seules qui permettent d'objectiver les récits de stratégies et de déceler des processus s'étendant sur un long terme, les chercheurs doivent souvent se contenter de narrations journalistiques ou d'analogies incertaines " (LANGE, 1986: 41). 
2002: p. 195) latino dès 1979, s'inscrit dans des processus - économiques, géopolitiques et idéologiques - plus larges tenant lieu à la " globalisation ". Si nous n'avons pas la place de les traiter en profondeur ici, nous pouvons d'ores-et-déjà souligner qu'ils impactent un secteur musical qui, dans ce contexte, présente certaines spécificités, que nous voudrions en revanche privilégier.

Ces spécificités se retrouvent notamment dans la stratégie hégémonique entreprise de longue date par l'industrie musicale étasunienne. L'implantation de filiales locales dans les divers pays latino-américains dès le début des années 1940 incite les acteurs dominants du secteur à la création de bureaux à Miami pour la gestion de ces marchés dits " régionaux ». L'émergence de Discos CBS International en 1979 à Coral Gables (ville située dans le comté de Miami-Dade) va par exemple en ce sens. Intégrée au sein du groupe Sony Music Entertainment en 1988, elle est une première étape dans la stratégie transnationale qu'entreprend la major à ce moment-là. La ville devient une nouvelle plaque-tournante des artistes et contenus culturels latino, par le recours constant au réseau de filiales dont elle dispose entre les deux parties du continent. Ce sont là des éléments problématiques que nous nous proposons d'approfondir, dans un premier temps d'un point de vue théorique.

\subsection{Une approche socioéconomique des industries culturelles}

Les stratégies d'intégration du capital dans les industries de la culture et de la communication représentent une des en- trées importantes de l'analyse des industries culturelles. Certaines études, comme celles menées en France par Philippe Bouquillion, montrent comment le système capitaliste s'est progressivement étendu à ces industries, qui, par intégration au sein de la sphère marchande, occupent désormais une place prépondérante dans le " centre " de l'économie mondiale (BOUQUILLION, 2008). Les stratégies économiques, qui y sont analysées avec précision, conduisent au constat d'une concentration oligopolistique croissante à partir des années 1980, constituée de fusions et acquisitions ininterrompues. Ces différentes formes d'intégrations verticales et horizontales mènent à une limitation de plus en plus prégnante de la concurrence. Celle-ci favorise les grands groupes transnationaux tout en contribuant, sur fond de mondialisation, à une centralisation du capital, comprise comme une « réduction du nombre des centres de décision "indépendants" " (BOUQUILLION, 2005: p. 113). Ces éléments rejoignent ainsi les travaux sur les villes globales menés depuis le début des années 1990 (SASSEN, 1991; GROSFOGUEL, 1995), qui montrent la manière dont certaines métropoles, à l'image de Londres, Tokyo ou New York, sont devenues des " nœuds centraux d'une nouvelle économie de service internationale " (SASSEN, 1996). Capables d'opérer à toutes les échelles un rôle stratégique de commandement et d'être dominantes en termes de transactions financières - ce que les nouvelles technologies de l'information et de la communication (NTIC) ont rendu possible -, ces villes globales seraient devenues des lieux centraux

3. Nous nommons " majors " les grandes compagnies de production transnationales se trouvant en position dominante dans le secteur de la musique. Leur nombre a évolué au fil des multiples fusions et acquisitions qui ont débuté aux États-Unis dans l'entre-deux-guerres, pour se retrouver aujourd'hui concentrées autour de trois principaux acteurs : Universal Music Group, Sony Music Entertainment et Warner Music Group. 
de concentration des richesses mondiales et de polarisation des flux, en particulier économiques et migratoires. Ainsi, ces approches invitent à penser les flux et circulations - tant économiques, migratoires que culturelles - selon différentes échelles.

\subsection{Une prise en compte d'échelles mul- tiples}

Bernard Miège, chercheur pionnier de la théorie des industries culturelles en France, explique dans un article portant sur le courant de l'économie politique de la communication (EPC) l'importance de l'articulation entre les dimensions " macro-, meso- et micro- ", souvent absente de ce type d'approche (MIĖGE, 2004). Or, la prise en compte de ces différentes échelles est indispensable à l'analyse de l'industrie musicale internationale, car elle permet de dépasser le simple binarisme global/local. Il ne s'agit plus dès lors de penser les deux extrêmes en miroir l'une de l'autre, mais au contraire l'entre-deux, zone d'ombre où s'élaborent les processus dynamiques de passage du local au global. L'ensemble des rouages se situant entre les stratégies " d'inclusion globale et de distinction locale " (GRASSY; SKLOWER, 2015: p. 41) peuvent ainsi être reconstitué. Peter Dicken, géographe économique, soutient dès 1986 la nécessité de ne pas considérer uniquement ces deux pôles d'observation :

C'est une erreur de se concentrer uniquement sur les deux extrêmes de l'échelle - le global et le local - où les activités économiques prennent place. Il est plus réaliste de penser en termes d'échelles d'activité et d'analyse reliées entre elles : par exemple, le local, le national, le régional et le global (DICKEN, 1986. In: LECLER, 2013).
Or, comme nous le disions dans l'introduction, à partir des années 1980 des chercheurs se sont attelés à décrypter la structuration de l'industrie musicale internationale, de plus en plus concentrée autour de grands acteurs en situation oligopolistique. Si les rapports de pouvoir qui se jouent entre les majors et les plus petites structures nationales sont décrits avec précision, tout comme les risques que cela fait porter à la diversité musicale et plus largement culturelle, c'est la prise en compte de ces enjeux, situés entre le local et le global, qui semblent primordiaux dans leur analyse des musiques populaires, dès lors caractérisées par leur circulation.

\section{L'industrie musicale: entre le local et le global}

\subsection{André Lange et le cas du marché belge francophone}

André Lange, dans son ouvrage intitulé Stratégies de la musique, est un des premiers à penser les rapports de la création musicale locale avec les logiques marchandes des grandes firmes multinationales (LANGE, 1986). Il y décortique, au travers de nombreux exemples tirés du territoire belge francophone, les articulations - verticales - entre l'oligopole dominant et les acteurs locaux. Un double processus caractérise selon lui ces articulations. D'une part, les industries phonographiques cherchent à importer un catalogue international dans des marchés nationaux, en s'appuyant sur une stratégie d'implantation de filiales locales. Par ce biais, elles peuvent distribuer et donc valoriser économiquement, à grande échelle, des contenus musicaux produits dans d'autres territoires. 
D'autre part, pour ne pas " asphyxier totalement les cultures nationales des pays où elles s'implantent " (LANGE, 1986: p. 40$41)^{4}$, elles font progressivement appel aux ressources locales. Cela passe par le développement d'artistes locaux qui ne seront valorisés, pour la grande majorité, que sur le marché d'où ils proviennent, prenant ainsi part à l'essor d'un répertoire local. Si certains artistes, selon des critères précis, peuvent être récupérés pour être distribués à une échelle internationale, la plupart restera dans ces marchés locaux ${ }^{5}$.

À travers cette circulation des artistes et répertoires selon différents niveaux (de promotion et de distribution), le rôle des structures indépendantes est alors réévalué, aux dépens d'une "surévaluation de la puissance des firmes constituant l'oligopole ". A. Lange insiste sur " la persistance, dans les interstices de l'immense toile d'araignée tissée par les multinationales, de firmes non monopolistes, de labels auxquels revient le rôle novateur et stimulateur " (LANGE, 1986: p. 83). Pour autant, il ne tombe pas dans une vision romantique d'une opposition entre " éditeurs artisans " et " éditeurs industriels ", où les premiers s'intéresseraient uniquement à la culture, les seconds à sa rentabilisation. L'opposition entre " monopole " et " non-monopole " rend selon lui mieux compte " des origines historiques de l'investissement de la musique par le capitalisme ". Ainsi, les articulations qu'il dé- crit entre ces deux types d'acteurs seraient l'autre lieu de l'articulation entre le local et le global. Celle-ci dépendrait à la fois des stratégies d'implantation des grandes entreprises multinationales, et des liens qu'elles entretiennent avec les acteurs indépendants sur ces territoires.

Néanmoins, à travers l'exemple du marché belge, il rappelle à quel point le déséquilibre est grand entre l'importation (du répertoire international) et l'exportation (du répertoire local), et l'ampleur de l'inégalité du rapport de force dans la distribution musicale entre firmes multinationales et organisations indépendantes. Tous ces éléments conduisent donc à " une hégémonie des produits anglo-saxons sur le marché belge " (LANGE, 1986, p. 337) et, pour aller plus loin, " à l'émergence d'une esthétique globalisée hégémonique " (DA LAGE ; DEBRUYNE, 2013).

\subsection{Robert Burnett et la domination des contenus étasuniens}

Cette critique se retrouve au cœur de l'ouvrage de Robert Burnett, The Global Jukebox, portant sur l'industrie musicale internationale (BURNETT, 1996). S'il y rejette la proposition de Jeremy Tunstall selon laquelle " les médias sont Américains " (TUNSTALL, 1977), la question de l'hégémonie des contenus étasuniens sur des marchés dorénavant globalisés est largement

4. André Lange reprend ici la thèse développée par Armand Mattelart selon lequel « les multinationales n'asphyxient jamais totalement les cultures nationales des pays où elles s'implantent, pour mieux assurer la dépendance par rapport à l'impérialisme américain ".

5. Cela dit, il rappelle que certains des artistes produits dans les différents marchés locaux, par leur " différenciation locale ", peuvent apparaître " comme un stimulant à l'achat du marché international ". Le rock en est, selon lui, un bon exemple : " il s'agit en effet du genre musical par lequel les multinationales (C.B.S., Warner) ont réussi leur implantation sur le marché mondial, et c'est en même temps le genre dont les filiales des multinationales encouragent le développement local ", p. 147. 
traitée. Dans le même temps, il articule cette analyse de la domination d'un petit nombre d'entreprises transnationales sur le marché mondial, à une étude sur l'émergence de musiques locales à l'échelle globale.

Il commence par expliquer que l'industrie de la musique étasunienne et britannique, avant la guerre, tirait l'essentiel de ses profits des marchés domestiques. Les marchés étrangers ne représentaient donc pas une part suffisamment importante de leurs revenus pour adapter les contenus aux marchés locaux ou pour essayer d'y maintenir un réseau de filiales élaboré. Mais, dans un monde où " les marchés, et en particulier l'économie culturelle, sont de plus en plus globalisés ", la situation change. Les grandes entreprises développent une logique transnationale afın d'intégrer massivement les marchés étrangers, par toute une série de processus économiques (" acquisitions, fusions, accords avec d'autres compagnies, voire disparition de concurrents ", BURNETT, 1996: p. 13), jusqu'à atteindre un taux de concentration ne laissant que peu de place aux acteurs locaux. Dans ce nouveau contexte, les industries musicales étasuniennes et britanniques tirent dès lors plus de la moitié de leurs revenus de ces marchés étrangers, en même temps que " les phonogrammes américains comptent pour plus de la moitié des ventes mondiales de musique enregistrée " (BURNETT, 1996: p. 4). Même si les analyses autour de l'» impérialisme américain " peuvent être considérées selon lui comme n'étant plus d'actualité (du simple fait que les majors sont désormais autant étasuniennes que japonaises ou européennes), la question de l'homogénéisation de la production des contenus culturels consommés à une échelle globale continue de se poser.

Parallèlement à cette analyse générale de la production et de la consommation de contenus culturels à l'échelle globale, Robert
Burnett affine l'articulation entre le local et le global autour d'une étude de cas. Celleci porte sur la " musique pop suédoise ". En partant de l'exemple d'ABBA, il montre comment toute une " scène musicale suédoise " a progressivement réussi à atteindre une audience mondiale, à mesure que les compagnies transnationales prenaient le contrôle de l'industrie musicale suédoise.

Ainsi, dix ans après l'ouvrage d'André Lange, il met en exergue la double stratégie des majors de l'industrie du disque sur les marchés locaux : jusqu'alors principalement impliquées dans la distribution de leurs catalogues sur ces territoires et dans la production d'artistes destinés au marché domestique, elles semblent de plus en plus capables de tirer profit de ces ressources culturelles locales. Cela passe par d'importants investissements et par des stratégies de valorisation (promotion, distribution) à l'échelle mondiale qu'elles seules peuvent mettre en place. En conséquence, si les médias ne sont plus américains, " ils n'en participent pas moins à la création d'une esthétique globalisée largement dépendante des conditions économiques de sa production " (DA LAGE ; DEBRUYNE, 2013).

\subsection{Nestor García Canclini et la mise sous tutelle étasunienne du marché lati- no-américain}

Enfin, un autre chercheur critique, l'anthropologue argentin Nestor García Canclini, s'est intéressé à l'articulation entre le local et le global dans le champ des industries culturelles. Son approche semble particulièrement heuristique pour notre sujet car elle questionne les rapports de domination interaméricains dans le secteur de la culture populaire. Spécialiste de l'étude des politiques culturelles, il ajoute ainsi cette dimension 
dans l'analyse de la circulation des contenus culturels à une échelle régionale et mondiale.

$\mathrm{Au}$ cours d'une recherche portant sur l'économie et la culture " des pays latins dans la sphère publique transnationale " (GARCÍA CANCLINI, 2001), N.G. Canclini montre comment " l'industrie de la musique, qui n'a cessé de se concentrer au sein de grands conglomérats transnationaux depuis l'invention de la musique enregistrée, prend une place toujours croissante dans notre économie "globale" ". À travers ce secteur, il analyse les liens et rapports de force entre les États-Unis et l'Amérique latine, et en vient à formuler une hypothèse sur la position hégémonique de l'un sur l'autre. Alors qu'il en arrive au même constat que Robert Burnett selon lequel " les États-Unis sont les principaux bénéficiaires de l'expansion économique et communicationnelle favorisée par les industries culturelles ", il se demande pourquoi le marché latino-américain, " qui connaît les taux de croissance les plus hauts depuis les années 1990 " et dont le dynamisme n'est plus à prouver, " autant en termes de public potentiel que de masse productive ", " reste à la traîne des pays bénéficiaires " ? En effet, les États-Unis accaparent selon lui « $55 \%$ des revenus/bénéfices mondiaux, quand l'Union européenne en reçoit $25 \%$, le Japon et l'Asie $15 \%$ et les pays ibéro-américains seulement $5 \%$ ".

Surtout, il rappelle que $80 \%$ du chiffre d'affaires réalisé par l'industrie musicale latino-américaine revient aux entreprises étrangères, en grande partie étasuniennes. S'il ne développe pas les causes de ce constat - ce que nous allons faire ici en analysant la stratégie transnationale mise en place par l'industrie musicale étasunienne sur l'Amérique latine à partir des années $1940^{6}$-, il en expose clairement les conséquences :

En dépit du fait que la production locale constitue un apport considérable à ce qui se trouve sur le marché international de la musique, et de la nette préférence des publics pour ce qui est conçu dans leur propre pays ou en langue espagnole, la situation économique des sociétés latino-américaines ne s'en trouve pas pour autant améliorée (GARCÍA CANCLINI, 2001: 248).

Au-delà de l'industrie musicale seule, l'origine de cette domination des entreprises culturelles nord-américaines sur le marché latino-américain se trouve selon lui dans « l'absence de politique culturelle commune entre les différents pays d'Amérique latine ", ce qui favoriserait " sa capacité à tirer profit de ses productions locales sur le marché culturel mondial ". Ainsi, l'absence d'échanges et de cohésion politiques dans le domaine de la culture aurait conduit à une fragmentation du marché latino-américain, isolant des pays principalement focalisés autour d'enjeux nationaux. Les États-Unis auraient alors tiré profit de cette situation

6. Nous pourrions même faire remonter ces processus au début des années 1910. En effet, Gérard Borras et Fred Rohner montrent comment la Victor Talking Machine (producteur de disques phonographiques étasunien), entreprend à partir de 1913 l'enregistrement de musiques populaires péruviennes, pour conquérir, dans sa lutte face à Columbia, le marché latino-américain et, à terme, " créer un marché musical mondial ". Mais il s'agissait avant tout de productions enregistrées " sur le terrain " ou à New York (en faisant voyager les musiciens repérés sur place), et non pas - encore - via l'implantation de filiales locales (BORRAS; ROHNER, 2013: p. 10). 
en poursuivant une politique hégémonique sur l'autre partie du continent, entamée, elle, de longue date dans les champs politique et économique.

Dès lors, le chemin pour une installation durable des entreprises culturelles étasuniennes en Amérique latine est ouvert. Cependant, dans ce processus, Nestor García Canclini ne va pas jusqu'à montrer comment la ville de Miami en est venue à jouer un rôle prépondérant dans la gestion de ce marché latino-américain et, conjointement, du marché latino-étasunien ; ce dernier prenant une place croissante au sein du marché intérieur nord-américain.

À partir de ces analyses sur l'articulation entre le local et le global au sein de l'industrie musicale transnationale, nous allons donc montrer comment des rapports de domination se sont instaurés dans ce secteur entre les États-Unis et l'Amérique latine et la place croissante que la ville de Miami y a prise. C'est en développant très tôt une capacité d'articulation entre ces différentes échelles que les majors, de plus en plus via la ville floridienne, se sont imposées sur le marché dit latino ; ce dernier s'étant construit autour de la représentation hégémonique d'une identité panethnique qu'elle a elle-même contribué à élaborer.

\section{La construction d'un marché latino}

Nous avons précédemment relevé que les approches développées par Saskia Sassen ou Ramón Grosfoguel autour des villes globales/mondiales - dans le sillage des études pionnières de Braudel (1979), Wal- lerstein (1979) ou Friedmann (1986) - ont permis de mettre l'accent sur une nouvelle géographie des flux transnationaux, qui replace les villes au premier plan des réseaux de circulation économique, financier, migratoire et culturel globaux. Certaines grandes métropoles urbaines occuperaient, aux dépens des États, une position nodale dans la mondialisation, où se concentreraient capital et pouvoir décisionnaire. Si Miami n'est pas reconnue comme disposant des attributs lui conférant le statut de ville globale, la plupart des observateurs reconnaissent sa position centrale dans les échanges entre les deux Amériques, alors constituées de certaines des "fonctions de ville globale "(SASSEN, 2012) ${ }^{7}$. George Yúdice, un des premiers chercheurs à avoir analysé dans le détail les industries culturelles de Miami (et en particulier l'industrie musicale), explique ainsi :

Miami doit se considérer comme une ville globale à échelle régionale, [...] par l'accumulation d'une main-d'œuvre peu coûteuse (grâce à l'immigration) et la concentration d'une masse critique d'entreprises et de services interconnectés permettant qu'une ou plusieurs entreprises produisent et exportent de façon globale (YÚDICE, 1999: p. 214).

\subsection{Miami, centre névralgique de l'enter- tainment latino}

Les multiples flux migratoires que la ville a connus aux lendemains de la révolution castriste sont alors replacés au centre de la réflexion. L'arrivée de 620000 Cubains

7. Voir en particulier le chapitre 5 intitule "Issues and Case Studies in the New Urban Economy ", dans lequel elle analyse le cas de Miami : "The Development of Global City Functions : The Case of Miami ", p. 181-188. 
fuyant le régime entre 1959 et 1973 en est le symbole, auxquels il convient d'ajouter les 125000 " Marielitos " qui prennent part à l'exode dans les cinq mois d'ouverture accordés par Fidel Castro entre le 21 avril et le 26 septembre 1980. Ces populations cubaines auraient permis à Miami de se développer et d'attirer banques et capitaux, engendrant par là même un nouvel attrait pour une ville en plein essor démographique, économique et culturel.

Dans cet environnement favorable du début des années 1980, les industries médiatiques et culturelles trouvent à Miami un nouveau lieu d'enracinement particulièrement prometteur. Le phénomène prend de l'ampleur au cours des années 1990, lorsqu'un nombre important de grands médias audiovisuels hispanophones vient s'installer ou ouvrir des filiales à Miami afın de conquérir " massivement " ce marché-là. C'est le moment où la ville devient un réel marché audiovisuel.

Les deux principales chaînes en espagnol des États-Unis par exemple, Univisión et Telemundo, vont progressivement y faire migrer leurs locaux. Telemundo, après avoir eu son siège à Hialeah, une ville de la banlieue de Miami où la concentration d'u Hispaniques " atteint les 94,7 \% (U.S. CENSUS BUREAU, 2010) ${ }^{8}$, a annoncé en février 2016 relocaliser l'ensemble de ses branches (network, studios, international et digital) dans un seul et même bâtiment situé dans le comté de Miami Dade (12200 NW 25th St.) (RODRIGUEZ, 2016). Univi- sión, quant à elle, à défaut d'avoir son siège à Miami, y installe dans le courant des années 2000 l'ensemble de ses studios de productions, essentiellement de telenovelas (MATO, 2002). C'est également le cas de MTV Latin America, qui s'installe à Miami en 1993, avec à l'origine un signal unique en espagnol pour toute l'Amérique latine hispanophone ${ }^{9}$. Enfin, au même moment, chacune des majors de l'industrie du disque implante ou renforce sa filiale latino à Miami Beach (PolyGram, BMG, EMI, Sony et Warner). La ville devient alors le centre névralgique de l'entertainment latino.

Dans ce processus, les grandes entreprises culturelles se positionnent stratégiquement dans des marchés intérieurs et extérieurs potentiellement très rentables. En effet, les Hispaniques deviennent à la fin des années 1990 la première minorité ethnique du pays, représentant 12,5\% de l'ensemble de la population ; tandis que le marché de la musique en Amérique latine poursuit son ascension dans les années 1990 jusqu'à posséder la plus importante croissance mondiale trois années de suite (1994-1997) avec $11 \%$ d'augmentation de ses ventes (YÚDICE, 1999: p. 187). Les majors font alors de leur implantation locale un enjeu majeur de leur développement transnational dès le début des années 1980 .

Pour observer la construction de ce marché latino et la place progressive qu'y prennent les industries culturelles de Miami, nous allons à présent nous pencher sur le cas de Sony Latin Music. Cette maison

8. Nous reprenons ici le terme d'" hispanique " utilisé comme synonyme de "Latino " par le bureau de recensement depuis le précédent rapport de 2000.

9. Depuis, un programme de régionalisation a été mise en place, consistant en une délocalisation de la programmation et de la production dans quatre grands pôles (MTV Norte à Mexico City en 1996, MTV Sur à Buenos Aires la même année, MTV Centro à Bogotá en 1999 ; MTV Brasil à São Paulo existant depuis 1990 avec un signal propre en portugais) afın d'accroître la pertinence locale d'une chaîne en perte d'audience. 
de disques a en effet été précurseur dans la mise en place d'un réseau de filiales en Amérique latine. Surtout, son rôle a été déterminant dans l'internationalisation $\mathrm{du}$ Miami sound et dans l'élaboration d'une stratégie multiscalaire pour y parvenir. Dès lors, elle devient un terrain d'étude pertinent pour notre analyse de la circulation de la musique latino mainstream ${ }^{10}$.

\subsection{Une politique précoce de rachat de maisons de disques en Amérique latine}

L'implantation dans la ville floridienne d'un centre de gestion pour le marché latino (rappelons que nous entendons par là sud-américain, centraméricain, caribéen et latino-étasunien) est le résultat d'un processus lancé dans les années 1940, quand le marché de la musique enregistrée en est encore à ses balbutiements.

À ses débuts en 1946, Sony se lance dans l'électronique et les télécommunications au Japon mais n'a aucune prétention dans la production et la distribution musicale. Elle se positionne véritablement sur ce secteur par le biais d'un investissement aux ÉtatsUnis lorsqu'elle met en place en 1968 une joint-venture avec CBS Records (à hauteur de 50/50), débouchant sur une acquisition totale en 1987. Sony bénéficie alors de la stratégie jusque-là mise en place par CBS Records, qui, depuis les années 1940, élaborait un réseau de filiales dans les principaux marchés latinoaméricains (SONY MUSIC ENTERTAINMENT, 2009) : au Mexique (1946), en Argentine
(1948), au Costa Rica qui dirige les marchés centraméricains (1962), en Colombie (1964), au Venezuela (1970), au Brésil (1971) et au Chili (1986) (YÚDICE, 1999: 192).

Surtout, la major décide de faire de CBS Discos International à Miami - renommée Sony Discos en 1991 - son centre des opérations pour, d'une part, l'Amérique latine et les Caraïbes (où elle poursuit cette stratégie transnationale en établissant des bureaux en Équateur en 1994, en Uruguay et au Pérou en 1996) et, d'autre part, pour le marché latinoétasunien en pleine croissance. Alors installés depuis 1979 à Coral Gables (dans le comté de Miami-Dade), les locaux sont déplacés à Miami avec l'arrivée d'un nouveau président en 1987, au moment où la maison de disques est totalement absorbée par le géant japonais. Lorsque Frank Welzer en prend les rênes, les ventes ne représentent que 6 millions de dollars par an (BILLBOARD, 1999, p. 58) et s'appuient en grande partie sur les revenus générés par les albums de Julio Iglesias, puis du Miami Sound Machine.

\subsection{Julio Iglesias et les prémices d'une stratégie multiscalaire}

Lorsque Julio Iglesias s'installe à Miami en 1979, Dick Asher le signe dans un double objectif : d'une part, il souhaite accroître l'internationalisation de ses ventes ; d'autre part, lui faire intégrer le marché nord-américain, et plus largement anglophone. Jusque-là, le chanteur espagnol a enregistré des albums en français, en allemand, en portugais et en

10. Le terme mainstream nous sert à désigner des " productions culturelles partagées par le plus grand nombre ", c'est-à-dire " largement diffusées dans la société " (DJAVADZADEH, RABOUD, 2016). En ce sens, elles peuvent être considérées, en suivant Frédéric Martel, comme "l'inverse de la contre-culture, de la subculture, des niches " (MARTEL, 2011). Toutefois, dans la perspective suivie par Stuart Hall, il ne faut pas voir le mainstream comme " un simple produit industriel de masse, mais un espace où s'expriment des tensions historiques sociales entre hégémonie et contre-hégémonie " (DJAVADZADEH, RABOUD, 2016). 
italien, vendus à plus de 12 millions d'exemplaires à travers l'Europe entière. L'accord conclu permet à la maison de disques de récupérer tous les droits des productions de l'artiste, y compris le catalogue existant dans la plupart des territoires. On comprend alors pourquoi Frank Welzer explique dans l'interview accordée à Billboard qu'» en 1979, c'était presque la compagnie de Julio. Plus de la moitié des ventes de la société provenaient du catalogue et des nouvelles sorties de Julio. Elle s'est constituée autour de lui ".

La stratégie commerciale mise en place par CBS International autour de Julio Iglesias est un premier jalon dans l'approche transnationale qui caractérisera par la suite l'industrie musicale de Miami, où il s'est agit de : conserver le marché européen ; s'étendre en Amérique latine grâce aux filiales locales de CBS ; et enfin développer le marché anglophone avec des chansons en anglais.

Ce triple objectif est atteint dès 1981 avec l'album hommage à sa fille De niña a mujer. Celui-ci obtient 130 disques d'or et 45 disques de platine à travers le monde (BILL$B O A R D, 27$ mars 1982). Il se place en tête des charts (palmarès de musiques enregistrées) en Europe (France, Italie, Espagne, Allemagne, Pays-Bas, Finlande) et en Israël. Comme prévu, le marché "Anglo " ${ }^{11}$ est intégré grâce à l'insertion de titres bilingues, où le refrain est chanté en anglais et les couplets en espagnol (" Begin The Beguine/Volver A Empezar ", " Island In The Sun/Isla En El Sol "). L'album se place alors numéro 1 des ventes en Angleterre. Enfın, l'objectif latino-américain est atteint, puisque De niña a mujer se trouve en tête des ventes dans les principaux marchés de la région, à savoir l'Argentine, le
Mexique, le Venezuela, la Colombie, le Chili et en Amérique centrale.

Le marché étasunien n'est quant à lui vraiment conquis que trois ans plus tard, en 1984, avec l'album 1100 Bel Air. D'importants moyens sont mis en place pour atteindre les foyers nord-américains. La majorité des titres sont en anglais et deux duos sont introduits avec des artistes de renom. La première chanson, "All of you ", est interprétée aux côtés de Diana Ross. La dernière, "To All The Girls I've Loved Before ", en compagnie du chanteur compositeur de musique country Willie Nelson. Ces deux artistes permettent d'englober une majeure partie des publics nordaméricains, incarnant des répertoires divers mais finalement complémentaires en termes de ressources exploitables. Trois millions de copies se vendent et positionnent le chanteur espagnol comme véritable star d'une culture populaire désormais mondialisée.

Le point fort de Sony Discos à Miami émerge à travers le cas précurseur de Julio Iglesias. Les possibilités ouvertes par le réseau de filiales, l'expertise des employés de la maison de disques dans la connaissance des multiples marchés, et la localisation géographique de Miami permettent d'insérer Julio Iglesias dans les marchés les plus importants en termes de revenus ou de potentialités : l'Europe (occidentale en particulier), les États-Unis et l'Amérique latine.

Dans une logique de circulation inverse, la maison de disques entreprend également la distribution de certains des artistes latino-américains les plus reconnus (Vicente Fernández et Roberto Carlos entre autres), afın de nourrir le marché latino-étasunien, qu'elle participe largement à constituer.

11. "Anglo " est une abréviation très utilisée dans le secteur musical pour évoquer le marché anglo-saxon/ anglophone dit mainstream, c'est-à-dire, dans ce cas, " dominant ". 
Enfin, Sony Discos est la première maison de disques de Miami à développer des artistes locaux destinés au marché latino dit mainstream. Encouragée par une stratégie ayant prouvé son efficacité avec Julio Iglesias, elle signe Willy Chirino (issu de la première génération de réfugiés cubains et considéré comme l'une des plus importantes stars musicales de Miami), sa femme Lissette et le Miami Sound Machine. Ce dernier groupe sort rapidement Sony Discos de sa "dépendance " envers Julio Iglesias. En effet, dès 1979, le label signe le quatrième album du Miami Sound Machine de Gloria et Emilio Estefan, peu de temps après sa formation et ses premiers albums produits dans des labels indépendants de la ville.

Par ces éléments, la maison de disques contribue donc à l'émergence d'un premier "Miami sound " sur le devant de la scène internationale tout en participant à la construction de l'image " latine " de la ville. Elle s'institue également comme première maison de disques latino des États-Unis. Même si au départ son rôle se limite à la distribution, le siège, installé à New York, lui accorde plus de libertés au milieu des années 1980, à mesure qu'elle prend conscience du potentiel du marché latino-étasunien.

\section{Conclusion}

L'on voit donc bien comment l'industrie dominante, à travers jeux et stratégies d'échelles, a constitué, depuis Miami, un secteur latino de poids sur le marché musical international. En 2005, celui-ci atteignait son pic avec 750 millions de dollars de revenus aux États-Unis. S'il est certes retombé en 2015 à 140 millions (RIAA, 2016), son potentiel reste grand aux vues de la croissance ininterrompue du marché latino-américain, qui demeure le marché régional le plus dyna- mique en termes de ventes pour la cinquième année consécutive, grâce à une augmentation annuelle de 11,8 \% (IFPI, 2016).

La stratégie ayant permis cette réussite, illustrée à travers l'exemple de CBS puis de Sony, pourrait se synthétiser par le processus dialectique suivant - déjà évoqué par Lange, Burnett et Canclini - : ouvrir à la fois un " marché de distribution ", par l'expansion des ventes dans les différents pays latinoaméricains, en développant de nouvelles filiales ou en rachetant les acteurs historiques des différents marchés phonographiques ; et un " marché de production ", en utilisant les multiples filiales nationales comme laboratoires pour faire émerger de nouveaux artistes permettant d'alimenter le marché local et global. L'investissement dans le développement de nouveaux artistes, au départ issus de Miami puis progressivement d'Amérique latine, sert donc bien ce double enjeu : développer les répertoires domestiques pour à la fois accroître l'emprise locale et dynamiser les ventes internationales.

Mais l'originalité de la stratégie du secteur latino se situe précisément dans la zone d'ombre que nous avons évoquée au départ : celle où se succèdent les différentes étapes du passage du local au global. Elle transparaît ainsi dans l'élaboration même des productions musicales, en se matérialisant par une sorte de " mise en générique ", à mesure que l'artiste évolue d'un marché à l'autre. Cette mise en générique prend racine dans une pan-latinité esthétiquement construite dans le but de dépasser les frontières (culturelles) nationales afin de toucher l'ensemble des publics latino (entendons par là latino-américains et latino-étasuniens) à une échelle régionale, puis l'ensemble des publics internationaux à une échelle globale. Nous pouvons dès lors affiner la description de la stratégie multisclaire, au tra- 
vers de trois grandes étapes - entrevues avec la trajectoire de Julio Iglesias - qui partent $\mathrm{du}$ local pour aller vers le national, le régional et enfin le global :

- En premier lieu, lorsqu'un nouvel artiste en provenance d'une des filiales latinoaméricaines est signé à Miami, la major cherche d'abord à atteindre les publics latino-américains avec des albums aux textes uniquement en espagnol.

- Dans un second temps, elle implante l'artiste aux États-Unis, d'abord par le biais de la communauté hispanophone avec des albums bilingues, puis sur le marché nord-américain dans son ensemble en insérant des paroles majoritairement en anglais. C'est ce qu'on appelle l'étape du " crossover ", qui a par ailleurs été analysée dans une perspective critique (CEPEDA, 2003; GAROFALO, 2005, 2010), en pointant les rapports de force établis entre cultures minoritaires et culture dominante blanche anglo.

- Enfin, lorsque l'artiste dispose d'une notoriété importante, il est propulsé sur le marché international grâce à la puissance du réseau de distribution de la major et un plan marketing d'ampleur qui, lui, s'inscrit dans une logique d'adaptation aux diverses audiences visées. Dans cette dernière étape, une identité (panethnique) latino est fortement valorisée afın de se distinguer de la pop internationale qui inonde déjà les circuits médiatiques mondiaux.

$\mathrm{Si}$ nous nous sommes appuyés sur l'exemple particulier de Sony pour expliciter cette stratégie, notre travail doctoral (BÉNISTANT, 2016) nous permet de dire qu'elle s'est très rapidement généralisée aux autres filiales des majors présentes à Miami. Celles-ci comprennent PolyGram Latino (qui deviendra Universal Music Latin Entertainment), BMG U.S. Latin (qui fusionnera avec Sony Norte en 2004), Warner Music Latina et EMI Latin. Elle a dès lors permis de faire émerger sur le devant de la scène internationale un grand nombre d'artistes de la culture populaire latino-américaine, tels que Shakira (Colombie), Ricky Martin (Porto Rico), et plus récemment Chino \&t Nacho (Venezuela), Calle 13 (Porto Rico) ou Nicky Jam. Ce dernier chanteur de reggaeton portoricain fait l'objet d'un article dans le rapport 2016 de l'IFPI sur le marché mondial de la musique : " Nicky Jam. Taking Latin Music to the World ". Les deux premiers paragraphes, auxquels nous renvoyons le lecteur (ci-dessous), concluent à merveille notre propos, en exposant d'emblée le modèle dépeint tout au long de cet article et qui, manifestement, a de beaux jours devant lui.

Figure 1 - Nicky Jam. Taking Latin Music to the World, Global Music Report, IFPI, 2016, p. 30.

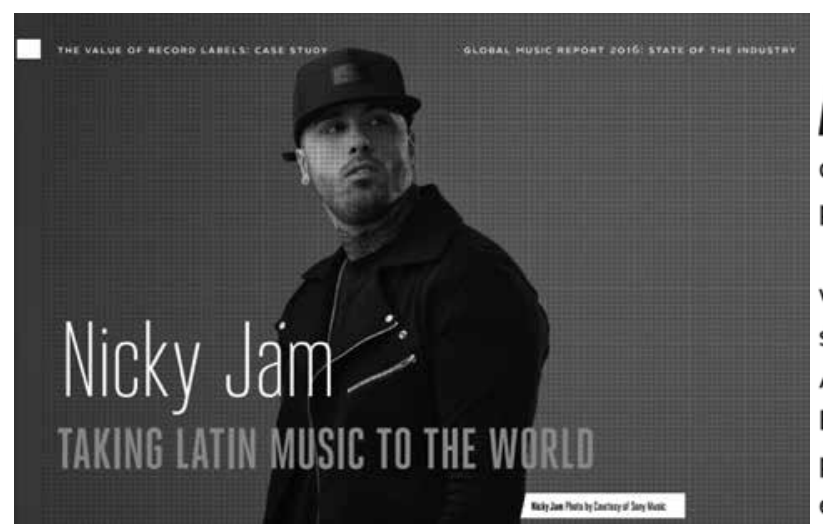

I Perdon by Nicky Jam and Enrique Iglesias demonstrates the power of Latin music to transcend international borders, as well as the role of the record company in helping make that possible.

The Colombia-based Reggaeton singer, who is signed to Sony US Latin, had enjoyed success for more than a decade within Latin America. But it was his collaboration with Enrique Iglesias on the song El Perdon that propelled him on to an international audience outside of Latin America. 


\section{Bibliographie}

BÉNISTANT, A. De la naissance du Miami sound: logiques de transnationalisation et de territorialisation de l'industrie musicale latino. Thèse de doctorat sous la direction de Tristan Mattelart, Université Paris 8, CEMTI, Saint-Denis, soutenue le 16 novembre 2016.

BILLBOARD. " Interview de Frank Welzer ", dossier spécial 20e anniversaire de Sony Discos (1979-1999), 20 novembre 1999, p. 58.

BORRAS, G.; ROHNER, F. La música popular peruana. Lima-Arequipa/ 1913-1917: los archivos de la victor talking machine. Lima: Instituto de etnomusicología, Instituto de estudios andinos, 2013.

BOUQUILLION, P. Les industries de la culture et de la communication: les stratégies du capitalisme. Grenoble: Presses universitaires de Grenoble, 2008.

BOUQUILLION, P. La constitution des pôles des industries de la culture et de la communication. Entre "coups" financiers et intégration de filières industrielles. Réseaux, vol. 3, n. 131, p. 111-144, 2005.

BRAUDEL, F. Civilisation matérielle, économie et capitalisme, XVe-XVIIIe siècle 1. Les Structures du quotidien - 2. Les Jeux de l'échange - 3. Le Temps du monde. Paris: Armand Colin, 1979.

BRIGHT, T. Pop Music in the USSR. Media, Culture and Society, vol. 8, p. 357-369, 1986.

BURNETT, R; WEBER, R. P. Concentration and diversity in the popular music industry 1948-1986. Annual meeting of the American Sociological Association in San Francisco, 1989.

BURNETT, R. The Global Jukebox: the International Music Industry. Londres: Routledge, 1996.

CEPEDA, M.E. Mucho Loco for Ricky Martin: or The Politics of Chronology, Crossover, and Language within the Latin(o) Music "Boom". In: BERGER, H.M.; CARROLL, M.T. (Orgs.) Global Pop, Local Language. Jackson: University Press of Mississippi, 2003, p. 113-130.
DA LAGE, E.; DEBRUYNE, F. Musique et perspectives critiques à l'ère des industries créatives. In: MATTELART, T. (Orgs.) Contributions aux recherches critiques sur la communication. Les Enjeux de l'information et de la communication, Supplément 2013, mis en ligne le 9 avril 2013, disponible en ligne : http://lesenjeux.u-grenoble3. fr/2013-supplement/06DaLageDebruyne/index. html [dernière consultation le 06/01/2017].

DICKEN, P. Global Shift: Mapping the changing Contours of the World Economy. Londres: Harper Et Row, 1986.

DJAVADZADEH, K; RABOUD, P. Le populaire estil soluble dans les industries culturelles ? Courants dominants et contraires des cultures populaires. Raisons politiques, vol. 2, n. 62, p. 5-20, 2016, doi:10.3917/rai.062.0005 [dernière consultation le 05/05/2017]

FRIEDMANN, J. The World City Hypothesis. Development and Change, vol. 17, n. 1, p. 69-83, 1986.

GARCÍA CANCLINI, N. Économie et culture: les pays latins dans la sphère publique transnationale. Trois espaces linguistiques face aux défis de la mondialisation. Paris: Organisation Internationale de la Francophonie, p. 241-256, 2001.

GAROFALO, R. Black popular music. Crossing Over or Going Under?. In: BENNETT et al. Rock and Popular Music. Politics, Policies, Institutions. New York: Routledge, 2005 [1993], p. 229-246.

GAROFALO, R. Rockin'out. Popular Music in the USA. Londres: Pearson, 2010.

GRASSY, E.; SKLOWER, J. Politiques des musiques populaires au XXIe siècle. Guichen: Éditions Mélanie Seteun, 2015.

GROSFOGUEL, R. Global logics in the Caribbean city system. The case of Miami. In: KNOX P.L.; TAYLOR P.J. (Orgs.) World Cities in a World-System. Cambridge, New York: University Press, p. 156-170, 1995.

IFPI. Global Music Report, 2016.

LAING, D. The Music Industry and the 'Cultural Imperialism' thesis. Media, Culture and Society, vol. 8, p. 331-341, 1986. 
LANGE, A. Stratégies de la musique: l'industrie internationale de la musique enregistrée et l'édition phonographique dans la Communauté française de Belgique. Bruxelles: P. Mardaga, 1986.

LECLER, R. Sociologie de la mondialisation. Paris: La Découverte, 2013.

MARTEL, F. Mainstream. Enquête sur la guerre globale de la culture et des médias. Paris: Flammarion, 2011.

MATO, D. Miami in the transnationalization of the Telenovela Industry: On territoriality and Globalization. Journal of Latin American studies, vol. 11, n. 2, p. 195-212, 2002.

MATTHEWS, J.; PERTICOZ, L. (Orgs.). L'industrie musicale à l'aube du XXIe siècle. Paris: Éditions L'Harmattan, 2012.

MIĖGE, B. La théorie des industries culturelles (et informationnelles), composante des SIC. Revue Française des Sciences de l'information et de la communication, vol. 1, n. 1, 2012, disponible en ligne: http://rfsic.revues.org/80 [dernière consultation le 05/05/2017].

MIEGE B. L'économie politique de la communication. Hermès, n. 38, p. 46-54, 2004.

RIAA. 2015 Year-End Industry Shipment and Revenue Statistics, 2016.

RODRIGUEZ, R. NBC Universal Telemundo building new \$250 million headquarters in MiamiDade. Miami Herald, 9 février 2016, Disponible en ligne: http://www.miamiherald.com/news/business/article59274258.html [dernière consultation le 04/05/2017].

SASSEN, S. The global city. New York, London, Tokyo. Princeton: Princeton University Press, 1991.

SASSEN S. La ville globale: New york, Londres, Tokyo. Paris: Descartes \&t Cie, 1996,

SASSEN, S. Introduire le concept de ville globale. Raisons politiques, vol. 3, n. 15, p. 9-23, 2004. Disponible en ligne: www.cairn.info/revue-raisons-politiques-2004-3-page-9.htm [dernière consultation le 06/01/2017].
SASSEN, S. Cities in a World Economy. Thousand Oaks: Sage, 2012.

SONY MUSIC ENTERTAINMENT. Anual Report. United States Securities And Exchange Commission, 2009, disponible en ligne : http:// www.sony.net/SonyInfo/IR/library/FY08_20F_ PDF.pdf [dernière consultation le 24/03/2016].

TUNSTALL, J. The Media are American: Anglo-American Media in the World. Londres: Constable, 1977.

WALLERSTEIN, I. The Capitalist World-Economy. Cambridge: Cambridge University Press, 1979.

YÚDICE, G. La industria de la música en la integración América Latina-Estados Unidos. In: GARCÍA CANCLINI, N.; MONETA J.C. (Orgs.) Las industrias culturales en la integración latinoamericana. Buenos Aires: LUDLBA/Mexico, Grijalbo SLLAUNESCO, 1999. 


\section{RÉSUMÉ}

Cet article interroge les processus qui interviennent dans le passage du local au global des contenus latino produits par l'industrie musicale de Miami. Comment un artiste dit " latino " intègre la culture populaire mainstream et quels sont les dispositifs (économiques, esthétiques) mis en place pour y parvenir ? En partant de certaines des études pionnières sur l'industrie musicale internationale, nous cherchons à mettre en lumière la stratégie multiscalaire élaborée par les labels dominants de Miami, investis dans le marché latino depuis la fin des années 1970, pour implanter des artistes successivement dans le marché national, régional et international.

\section{PALAVRAS-CHAVE}

Industrie musicale. Miami. Marchés latino. Local/global.

\section{ABSTRACT}

This article examines the processes involved in the transition from local to global, of Latin contents produced by the Miami music industry. How does a "Latin artist" integrate mainstream popular culture? What are the (economic, aesthetic) apparatus designed to achieve this? Starting from some of the pioneering international music industry studies, we highlight the multiscalar strategy developed by the Miami dominant labels to introduce new artists into national, regional and international markets.

\section{KEYWORDS}

Music Industry. Miami. Latin Markets. Local/global. 